\title{
高速切削中切削速度对工件材料力学性能和切屑 形态的影响机理
}

\author{
苏国胜 ${ }^{(1)}$ 刘战强 ${ }^{(2 *} ，$ 万熠 $^{2}$ ，艾兴 ${ }^{2}$ \\ (1) 山东轻工业学院机械与汽车工程学院, 济南 250353; \\ (2) 山东大学机械工程学院, 济南 250061 \\ *E-mail: melius@sdu.edu.cn
}

收稿日期: 2012-05-17; 接受日期: 2012-07-25

国家重点基础研究发展计划(“973”计划) (批准号: 2009CB724401)和山东省自然科学杰出青年基金(批准号: JQ200918)资助项目

\begin{abstract}
摘要 首先根据不同应变率阶段金属材料的塑性变形机制, 分析工件材料强度和塑脆性随切 削速度提高的变化规律, 通过理论计算说明这种变化对切屑锯齿化的作用机制, 揭示不同切削 速度下产生不同形态切屑的控制机理; 然后，在 40 7000 $\mathrm{m} / \mathrm{min}$ 切削速度范围对 Inconel718, AerMet100, 7050-T7451 进行直角切削实验, 分析切屑形态及工件材料脆化现象, 验证随切削速 度提高工件材料脆化及相应的切屑形态; 最后, 根据切削速度与切屑形态的对应规律, 提出基 于切屑形态的切削速度阶段划分方法. 研究表明: 随切削速度提高, 材料强度提高、塑性降低, 使得主变形区材料易于发生热塑性剪切失稳和断裂失效而使切首锯齿化; 对一般塑脆性金属材 料, 随切削速度提高, 第一变形区材料热塑性剪切失稳的发生早于断裂失效的发生; 对同一种 工件材料, 可根据切屑形态将切削速度划分为普通切削速度阶段、高速切削速度阶段、超高速 切削速度阶段.
\end{abstract} 关键词 高速切削 切屑形态 形成机理 材料动力学

切屑形态对切削过程中的排屑、刀具磨损、工件 已加工表面质量等具有重要的影响, 是进行工件材 料可切削加工性评价的重要方面. 周泽华教授 ${ }^{[1]}$ 将切 屑形态分为带状切屑、节状切屑(锯齿形切屑)、粒状 切屑(单元切屑)及崩碎切屑; Komanduri 和 Brown ${ }^{[2]}$ 将切屑形态划分为节状切屑、波浪形切屑、锯齿形切 屑及非连续切屑(单元切屑); 而 Trent 和 Wright ${ }^{[3]}$ 将切 屑形态划分为积屑瘤型切屑、连续切屑、锯齿形切屑 及非连续切屑. 上述波浪切屑和积屑瘤型切屑实质 上是带状切屑在特定切削速度阶段的切屑形态 ${ }^{[1,2]}$, 本质上同属于带状切屑. 而粒状切屑(单元切屑)、崩
碎切屑和不连续切屑只有大小区分, 本质上都是由 于切削层材料断裂失效形成的, 都属于不连续切屑 或称单元切屑. 因此切屑根据其形态可以划分为带 状切屑、锯齿形切屑和单元切屑三大类.

研究发现普通切削速度下切屑形态与工件材料 的力学和热物理性能间有重要的关系 ${ }^{[1,4,5]}$. 切削塑 性金属材料时容易形成带状切屑 ${ }^{[1]}$; 切削脆性金属材 料时容易形成单元切屑 ${ }^{[1,5 \sim 8,9]}$; 切削硬度高或热物理 性能差的金属材料时则容易形成锯齿形切屑 ${ }^{[10 ~ 13]}$. 带状切屑形成时, 由于材料的应变硬化效应, 主剪切 区已变形材料的硬度大于新进入剪切区材料的硬度,

中文版发表信息: 苏国胜, 刘战强, 万熠, 等. 高速切削中切削速度对工件材料力学性能和切屑形态的影响机理. 中国科学: 技术科学, 2012, 42: 1305-1317, doi: 10.1360/092011-533 
剪切变形被迫在新进入剪切区的材料内发生而形成 带状切屑, 切屑内发生了均匀的塑性变形 ${ }^{[1,4,14]}$. 如 果切屑沿剪切面滑移过程中, 剪切面上剪应力超过 了材料的抗拉强度, 则会沿剪切面断裂而产生单元 切屑 ${ }^{[1,5]}$. 锯齿形切屑是由于切削层材料流经第一变 形区时沿剪切面的塑性变形抗力突然降低导致的 ${ }^{[15]}$. 致使剪切面塑性变形抗力降低的原因是沿剪切面的 热塑性剪切失稳(或称绝热剪切失稳)或断裂失效. Nakayama 等人 ${ }^{[6]}$, Shaw 等人 ${ }^{[7]}$, Vyas 等人 ${ }^{[8]}$ 对锯齿形 切屑的实验研究表明: 淬硬钢或高强度钢锯齿形切 屑分节间存在断裂现象. 他们认为材料的高硬度致 使其塑性降低、脆性增加，切屑沿主剪切面滑出时， 塑性剪切变形演化为断裂而导致锯齿形切屑产生. Gente 等人 ${ }^{[13]}$, Băker 等人 ${ }^{[16,17]}$ 对热物理性能较差(即 $k \rho c$ 乘积值较低, 其中 $k$ 是材料热导率, $\rho$ 是材料密度, $c$ 是材料比热容)的钛合金的仿真和实验研究发现: 工件材料热物理系数低, 导致剪切变形做功产生的 热量来不及向外传导和辐射, 造成变形区材料温升 较高, 塑性剪切变形在较小应变时便因热软化效应 大于应变硬化和应变率硬化效应而失稳, 产生锯齿 形切屑.

同一工件材料在不同切削速度阶段也会产生不 同的切屑形态 ${ }^{[12,18,19]}$. 随切削速度提高, 一般塑性金 属材料的切屑形态典型演化过程为: 带状切屑 $\rightarrow$ 锯 齿形切屑 $\rightarrow$ 单元切屑. 研究发现, 随变形速度(应变 率)提高, 工件材料力学性能主要发生两个方面的变 化: 一是材料强度(屈服强度、抗拉强度)随应变率提 高而提高; 二是材料塑性随切削速度提高而下降, 特 别是当变形速度提高到一定程度后, 材料的塑性快

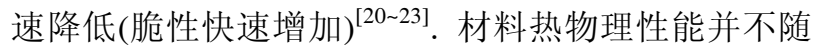
切削速度的提高发生明显变化, 因此随着切削速度 的提高材料强度和塑脆性的变化将对切屑形态产生 重要影响.

本文分析工件材料强度和塑脆性随切削速度的 变化规律, 探索工件材料强度和塑脆性变化对切屑 锯齿化的作用机理，阐述不同切削速度阶段产生切 屑的控制机制; 根据材料动态力学性能随切削速度 提高的变化规律, 对塑性金属材料切屑形态随切削 速度提高的演化过程进行理论预测, 并通过超高速 切削实验进行验证; 根据不同切削速度阶段切屑形 态不同的规律, 提出基于切屑形态的切削速度阶段 划分方法.

\section{1 切削速度(变形速度)对工件材料性能和剪 切变形失稳的影响}

切削过程中, 随切削速度提高, 第一变形区材料 应变率近似呈线性提高 ${ }^{[14]}$. 较高的应变率导致材料 的力学性能发生改变, 从而影响工件材料流经第一 变形区时的变形稳定性和切屑形态.

\section{1 变形速度(应变率)对工件材料强度和剪切失稳 的影响}

金属塑性变形是通过位错的滑移和增殖进行的, 材料晶格中的位错密度增大, 则需要更大的位错切 应力. 在较低应变率阶段 $\left(<10^{3} / \mathrm{s}\right)$, 控制塑性变形的 是热激活机制和由扩散控制的蠕变机制. 在该阶段 随变形速度的提高, 需更多的位错源同时开动, 结果 抑制了单晶体中位错易滑移阶段的产生和发展，使 材料晶格中位错密度和滑移系数目增加, 从而使材 料的临界屈服应力增大. 而当应变率 $\geqslant 10^{3} / \mathrm{s}$ 时, 位 错运动的控制机制是阻尼机制. 在该阶段, 应力高到 足以驱使位错越过所有的障碍而不需要任何热的帮 助, 位错来不及进行堆积和滑移, 晶格原子沿滑移面 同时翻越点阵阻力, 材料的屈服强度达到最高值 ${ }^{[20]}$. Clifton $^{[22]}$ 的冲击实验表明：当应变率达到 $10^{5} / \mathrm{s}$ 时 1100-0 铝合金等多种金属材料的屈服强度出现了快 速提高. 但不同塑性的材料, 其屈服强度具有不同的 应变率敏感性，毛卫民等人 ${ }^{[23]}$ 的研究结果显示：低 塑性材料如各种钢类, 随着变形速度提高材料的抗 拉强度增加较小, 而屈服强度增加较快, 而对于塑性 较好的材料, 其屈服强度变化不明显.

材料塑性变形开始后，变形区材料塑性变形做 功产生温升, 变形区材料热起伏、蠕变和动态再结晶 可使材料在较低的应力状态下产生塑性变形, 此即 是材料的热软化效应. 此时, 材料的流动应力由材料 应变硬化、应变率硬化和塑性变形做功引起的热软化 共同决定. 研究人员已建立了多个同时考虑应变硬 化、应变率硬化和热软化影响的材料模型 ${ }^{[24]}$, 其中 Johnson-Cook 材料模型 ${ }^{[25]}$ 因其结构简单、意义明确而 获得了广泛的应用, 其定义为

$$
\tau=\left(A+B \gamma^{N}\right) \times\left(1+C \ln \frac{\dot{\gamma}}{\dot{\gamma}_{0}}\right) \times\left[1-\left(\frac{T-T_{r}}{T_{m}-T_{r}}\right)^{M}\right],
$$

其中, $A$ 为屈服强度 $(\mathrm{Pa}) ; B$ 为应变强化系数; $N$ 为应变 
强化指数; $C$ 为应变率强化系数; $\gamma$ 为质点当前剪应变; $\dot{\gamma}$ 为质点当前剪应变率 $(1 / \mathrm{s}) ; \dot{\gamma}_{0}$ 为参考应变率 $(1 / \mathrm{s})$, 常取值为 $0.001 / \mathrm{s} ; T$ 为质点当前温度 $\left({ }^{\circ} \mathrm{C}\right) ; T_{r}$ 为室温 $\left({ }^{\circ} \mathrm{C}\right) ; T_{m}$ 为材料融化温度 $\left({ }^{\circ} \mathrm{C}\right) ; M$ 为热软化指数; $\tau$ 为质 点在当前应变、应变速率、温度下的流动应力 (Pa). (1) 式等号右侧的第一括号项反映材料的应变硬化效应, 第二括号项反映材料的应变率硬化效应, 第三括号 项反映的是材料的热软化效应. 给定一组应力、应变 率和温度数据可以由 Johnson-Cook 模型获得对应的 流动应力. 在变形过程中, 应变、应变率、温度之间 是相互耦合的: 应变、应变率的大小决定变形区温升 的高低, 而温升的高低又进一步影响流动应力的变 化和更进一步的温升. 在较高的变形速度下, 假设变 形区塑性变形做功产生的热量全部用于变形区材料 的温升, 即变形处于绝热状态, 则可以得到微分形式 表示的变形区温升计算式:

$$
\mathrm{d} T=\frac{\tau}{\rho C} \mathrm{~d} \gamma .
$$

塑性变形过程中, 变形材料的热-力耦合过程可 以采用图 1 的流程图实现.

随应变率提高, 材料的流动应力提高, 变形区的 温升和相应的热软化效应也提高, 这促使变形区内 的材料在更小的应变处发生热塑性剪切失稳. 以高 温合金 Inconel718 为例, Johnson-Cook 模型描述的

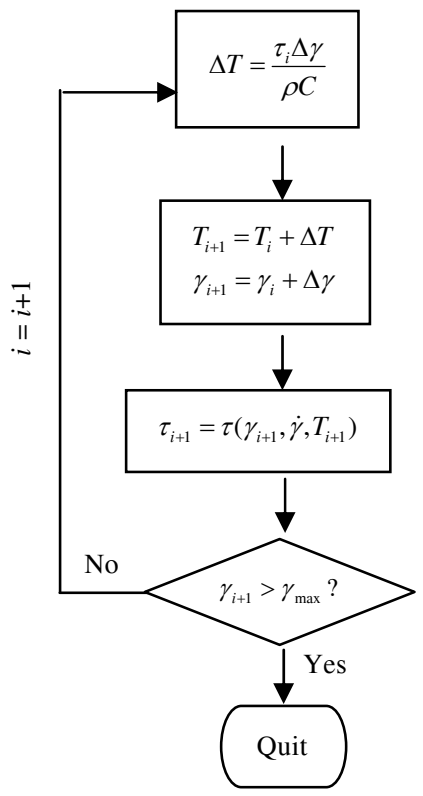

图 1 变形区材料热-力耦合过程的实现
Inconel718 材料在不同应变率下流动应力、温升和热 软化效应如图 2 所示. 为了对比不同应变率下的热力耦合效应, 图 2 中标识为 NTMC(No Thermal Mechanical Coupling)的曲线为不计热-力耦合时的应 力-应变曲线(图 2(a)) 和温度-应变曲线(图 2(b)), 而标 识为 TMC(Thermal Mechanical Coupling)的曲线为计 入热-力耦合时的应力-应变曲线(图 2(a))和温度-应变 曲线(图 2(b)). 计算所用 Inconel718 的 Johnson-Cook 材料模型常数如表 1 .

从图 2(a)NTMC 曲线可看出, 随应变率提高材料 的流动应力升高, 应变为 1 时, 应变率每升高 $\times 10^{3}$ 数量级, 流动应力提高约 $250 \mathrm{MPa}$. 在较大应变处, 应变率提高相同数量级, 流动应力提高也较高, 这是 由 Johnson-Cook 材料模型的定义形式决定的: Johnson-Cook 材料模型是在幂函数表示的材料应力应变关系((1)式第一项)上乘一个大于 1 的系数作为应 变率硬化效应, 所以第一项基数((1)式)较大时乘相同 的系数后产生的应力增量也就越大, 这与材料的变 形实际并不相符. 图 2(a)中 NTMC 曲线中是材料在静 态变形时 ( $\dot{\gamma} \leqq 0.001 / \mathrm{s})$ 的应力-应变曲线. 从图 2(b) $\mathrm{NTMC}$ 曲线可以看出, 较高的材料强度所引起的变 形区温升也较高. 应变为 1 时, 应变率每升高 $\times 10^{3}$ 数量级, 温度提高约 $50^{\circ} \mathrm{C}$. 温度的升高使流动应力 随应变的增加增速减缓, 且在一定的应变处流动应 力达到最大值并随后减小, 变形区材料发生热塑性 剪切失稳. 应变率越高变形区材料的热软化效应越 明显, 也使材料在更小的应变处发生剪切失稳：当 $\dot{\gamma}=1 / \mathrm{s}$ 时, 在 $\gamma=1.2$ 处发生剪切失稳; 当 $\dot{\gamma}=10^{3} / \mathrm{s}$ 时, 在 $\gamma=1.1$ 处发生剪切失稳; 而当 $\dot{\gamma}=10^{6} / \mathrm{s}$ 时, 在 $\gamma=0.9$ 处发生剪切失稳(图 2(a)TMC 曲线). 变形区温 升因材料的热软化而上升幅度变缓, 如图 2(b)TMC 曲线. 需要说明的是由于受限于实验方法所能达到 的应变、应变率的限制, 图 2 在较高应变率阶段的流 动应力和温度数据只能靠外推获得, 与实际流动应力 相比可能存在较大误差, 如在图 2 中无法反映出 Clifton 等人 ${ }^{[23]}$ 发现的当应变率大于 $10^{5} / \mathrm{s}$ 时材料流动 应力迅速升高的现象.

在金属切削过程中, 随切削速度提高第一变形

表 1 Inconel718 钢 Johnson-Cook 材料模型常数 ${ }^{[26]}$

\begin{tabular}{cccccc}
\hline$A(\mathrm{MPa})$ & $B(\mathrm{MPa})$ & $C$ & $N$ & $M$ & $\dot{\gamma}_{0}(/ \mathrm{s})$ \\
\hline 450 & 1700 & 0.017 & 0.65 & 1.3 & 0.001 \\
\hline
\end{tabular}



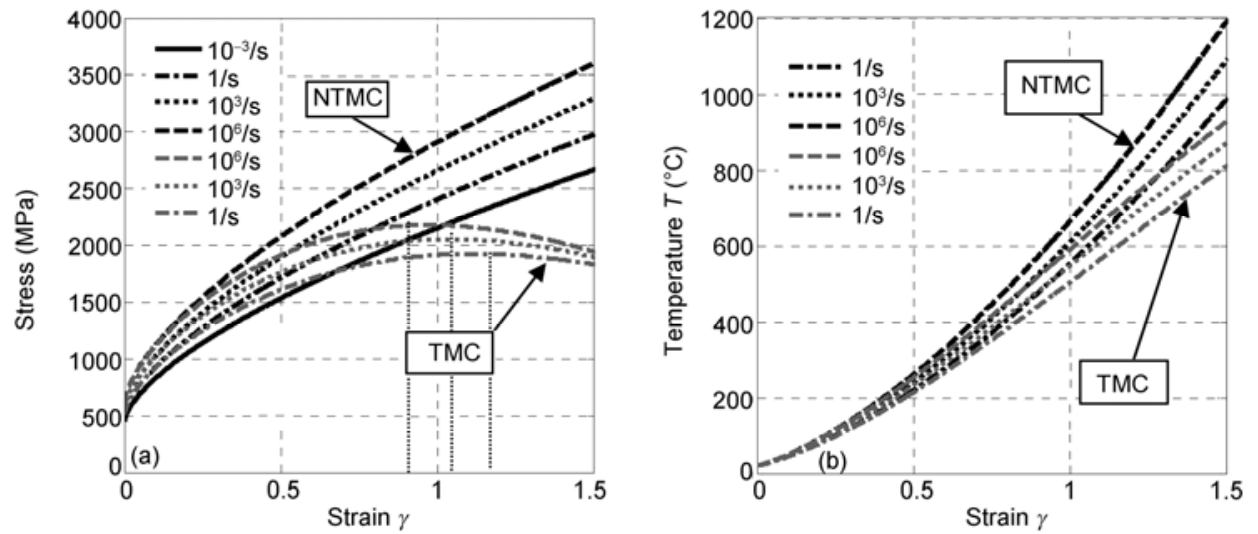

图 2 应变率对材料强度、变形区温升和热软化效应的影响

(a) 真实应力-应变曲线; (b) 温升-应变曲线

区材料的应变率近似呈线性增加 ${ }^{[14]}$. 在足够高的切 削速度下, 主剪切面上材料热软化引起流动应力的 下降大于应变硬化、应变率硬化引起流动应力的升高 时, 就会沿该剪切面发生热塑性剪切失稳, 并最终导 致锯齿形切屑产生. 不同切削速度下切削层材料流 经第一变形区时剪切失稳过程可以用图 3 说明. 图 3 中曲线 1 和 2 分别对应较低切削速度和较高切削速度 下, 切削层中某点 $\mathrm{A}$ 流经第一变形区时的应力-应变 演化曲线. 若 A 点流经第一变形区时的应力-应变关 系沿曲线 2 演化, 在未达到最大应变 $\gamma_{\text {max }}$ (材料断裂 时对应应变)前的 $\gamma_{i}$ 处 A 点发生热塑性剪切失稳(或断 裂), 则剪切面的剪切抗力下降, 剪切变形在 $\mathrm{A}$ 点所 处的剪切面继续发生而成为集中剪切带, 切屑成为 锯齿形切屑. 而若点 $\mathrm{A}$ 流经第一变形区时的应力-应 变关系沿曲线 1 演化, 在应变 $\gamma_{i}$ 处材料末出现应力下 降, 随点 $\mathrm{A}$ 继续进入剪切变形区, 点 $\mathrm{A}$ 处的应变继续 增加, 因应变硬化作用 $\mathrm{A}$ 点处的流动应力逐渐增大, 这就迫使剪切变形在新进入剪切变形区的材料内发 生. 在达到最大应变 $\gamma_{\text {max }}$ 前 $\mathrm{A}$ 点处流动应力没有出现 下降, A 点自 $\gamma_{\text {max }}$ 处流出第一变形区, 形成带状切屑. 因此, 基于热塑性剪切失稳的切屑形态由带状转变 为锯齿形的临界条件可以表达为

$$
\left.\frac{\partial \tau}{\partial \gamma}\right|_{\gamma \leqslant \gamma_{\max }} \leqslant 0 .
$$

可见, 随切削速度提高, 热软化效应增强, 变形 区材料发生剪切失稳时应变 $\gamma_{i}$ 将从 $\gamma_{\text {max }}$ 之外 (大于 $\gamma_{\text {max }}$ )

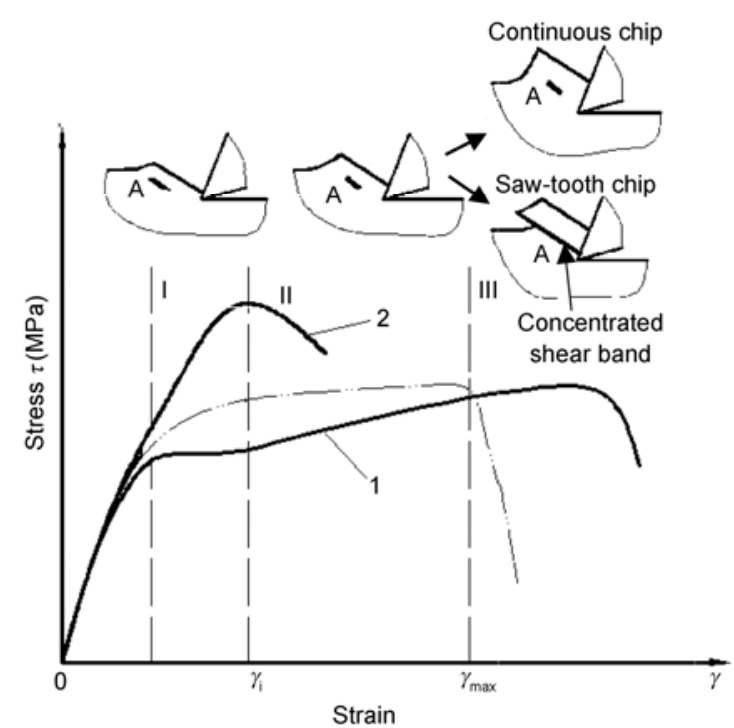

图 3 锯齿形切屑形成时的力学条件

移动到 $\gamma_{\text {max }}$ 之内 (小于 $\gamma_{\text {max }}$ ), 对应切屑形态由带状转变 为锯齿形. 在 $\gamma_{\mathrm{i}}=\gamma_{\max }$ 处, 对应切削速度就是该材料的 切屑锯齿化临界切削速度, 简称临界切削速度(本文 标示为 $V_{c r}$ ). 随切削速度(变形速度)提高, 材料强度 增高, 变形区材料的热软化效应增强, 这使得切削层 材料在较小的应变 $\gamma_{\mathrm{i}}$ 处发生热塑性剪切失稳, 从而促 进锯齿形切屑较早产生.

除材料强度变化对剪切失稳产生影响外, 随切 削速度提高变形区材料单位时间内产生的热量相对 于向外传导、辐射的热量增多, 此将加速材料的剪切 失稳过程. 这不是本文的重点, 在此不再展开论述. 


\section{2 变形速度(应变率)对工件材料塑脆性和剪切失 稳的影响}

材料在塑性变形时, 由于不同变形速度下变形 区温升和塑性变形控制机制不同，不同应变率阶段 材料的塑脆性也不同. 随变形速度提高金属材料塑 性整体呈现下降趋势 ${ }^{[20]}$. 在扩散蠕变机制控制阶段, 变形区材料温度较高, 高温高应力(应变率)下, 强烈 变形部位将迅速发生恢复再结晶, 晶界能够通过扩 散发生迁移, 从一定程度上弥补由于晶界滑动在晶 界处形成的空洞, 使材料的塑性得到提高. 随变形速 度的进一步提高, 变形部位恢复再结晶速度将晶界 处由于晶界滑动形成的空洞的速度, 沿晶界处空洞 迅速长大并最终断裂, 材料的塑性降低. 当位错运动 进入阻尼机制控阶段, 在此变形速度阶段位错来不 及进行堆积和滑移, 位错的移动以脆性断裂的形式 进行 ${ }^{[20,21]}$. Klepaczko 以不同的冲击速度对 XC18 钢进 行冲击剪切, 结果显示 (图 4(a)), 在应变率为 $10^{-3} \sim 10^{2} / \mathrm{s}$ 阶段, 工件材料的塑性缓慢提高; 然后随 应变率提高, 塑性开始出现缓慢下降; 而当应变率约 为 $10^{4.5} / \mathrm{s}$ 时 XC18 钢的失效应变快速下降(图 4(a)) ${ }^{[27]}$, 说明 XC18 钢在应变率为 $10^{4.5} / \mathrm{s}$ 时的塑性迅速降低, 脆性迅速增加. 材料屈服强度和抗拉强度随变形速 度提高的变化也能说明材料在高应变率阶段的脆化, 如 $40 \mathrm{CrNi}$ 钢静载荷时屈服强度和抗拉强度分别是 956 和 $1035 \mathrm{MPa}$, 在冲击速度为 $450 \mathrm{~m} / \mathrm{min}$ 时屈服强 度和抗拉强度分别是 1275 和 $1570 \mathrm{MPa}$, 在冲击速度 为 $600 \mathrm{~m} / \mathrm{min}$ 时屈服强度和抗拉强度都是 1187 $\mathrm{MPa}^{[20]}$, 即在冲击速度为 $600 \mathrm{~m} / \mathrm{min}$ 时 $40 \mathrm{CrNi}$ 变成了 脆性材料. 另外, 由于不同晶格金属具有滑移系的数 目不同，不同晶格的金属其塑脆性应变率敏感性不同， 具有 BCC 晶格的金属材料塑脆性对应变率较敏感, 而 FCC 晶格金属材料塑脆性对应变率不敏感 ${ }^{[28,29]}$.

金属材料的塑脆性不仅受材料变形速度、变形区 温度影响, 而且还受变形区应力状态影响 ${ }^{[30]}$, 其在宏 观上表现为材料的延展性, 在微观上可以表示为材 料断裂失效应变: 若失效应变大则说明材料的塑性 较好(脆性差), 失效应变小则说明材料的塑性差(脆 性好). 目前, 直接表达材料失效应变和应变率函数 关系式较少. Johnson-Cook 在 1985 年提出了一个同 时考虑变形区应变率、压应力状态和温度的等效失效 应变模型 ${ }^{[31]}$, 如下:

$$
\begin{aligned}
\varepsilon_{\mathrm{p}}^{\mathrm{f}}= & {\left[d_{1}+d_{2} \exp \left(d_{3} \frac{\sigma_{\mathrm{p}}}{\sigma_{\text {Mises }}}\right)\right] \times\left[1+d_{4} \ln \left(\frac{\dot{\varepsilon}_{\mathrm{p}}}{\dot{\varepsilon}_{0}}\right)\right] } \\
& \times\left[1+d_{5}\left(\frac{\theta-\theta_{\text {room }}}{\theta_{\text {melt }}-\theta_{\text {room }}}\right)\right],
\end{aligned}
$$

式中, $\varepsilon_{\mathrm{p}}^{\mathrm{f}}$ 为临界等效塑性失效应变, $\sigma_{\mathrm{p}}$ 为压应力 $(\mathrm{Pa})$, $\sigma_{\text {Mises }}$ 为 Mises 应力 $(\mathrm{Pa}), \quad \dot{\varepsilon}_{\mathrm{p}}$ 为应变率 $(1 / \mathrm{s}), \quad \dot{\varepsilon}_{0}$ 为参考 应变率 $(1 / \mathrm{s}), \theta_{\mathrm{melt}}$ 为材料熔化温度 $\left({ }^{\circ} \mathrm{C}\right), \theta_{\mathrm{room}}$ 为室温 $\left({ }^{\circ} \mathrm{C}\right)$. 根据文献 [32] 的数据, AISI4340 钢在室温 $\left(\theta=\theta_{\mathrm{room}}\right)$ 、纯剪切变形条件下 $\left(\sigma_{\mathrm{p}}=0\right)$, 失效应变 $\varepsilon_{\mathrm{p}}^{\mathrm{f}}$ 与 应变率的关系如图 4(b). 从图 4(b)可以看到, 随着应 变率的提高, AISI4340 失效应变单调提高, 说明在塑 性变形做功所产生热的作用下材料塑性增强. 但材 料失效应变在较高应变率阶段突然降低的现象(图 4(a)) 并没有在图 4(b)出现. Johnson-Cook 等效失效应 变模型能否描述材料塑性在较高应变率阶段突然降 低现象还有待于进一步研究. 目前, 材料塑性随变形 速度提高变化、特别是在高应变率阶段塑性降低的现 象, 还无法体现在当前各种基于连续介质假设的材 料本构方程中. Johnson-Cook 等效失效模型在切削问 题方面也仅用于高速切削过程的仿真研究: 即用(4) 式定义单元节点的等失效应变, 在变形计算时检测 变形区单元节点的等效应变, 若某单元节点的等效 应变等于或大于给定失效应变时, 将该单元力学属 性及对应网格删除, 以模拟工件材料断裂现象(这部 分具体内容可以参考文献[33, 34]).

从图 3 所示锯齿形切屑形成过程可知, 若沿穿过 A 点的剪切面发生断裂, 剪切面失去剪切变形抗力, 切屑沿断裂面向往滑出, 亦形成锯齿形切屑. 在较高 的切削速度阶段, 材料失效应变降低, 会使切削层材 料流经第一变形区时在较小的应变处沿剪切面断裂, 从而更容易产生锯齿形切屑.

\section{3 不同切削速度阶段切屑形态和切屑形成机理}

可见, 随变形速度提高, 材料强度的增加和脆化 都可以导致材料在较小的应变时失去塑性变形抗力, 从而使变形材料发生热塑性剪切失稳或断裂失效. 哪种剪切变形失稳机制起主导作用取决于当时的塑 性变形机制, 随变形速度提高, 热塑性剪切失稳要早 于断裂失效发生. 同时, 随变形速度逐渐提高, 材料 热塑性剪切失稳向断裂失效的过渡又是逐渐进行的. 

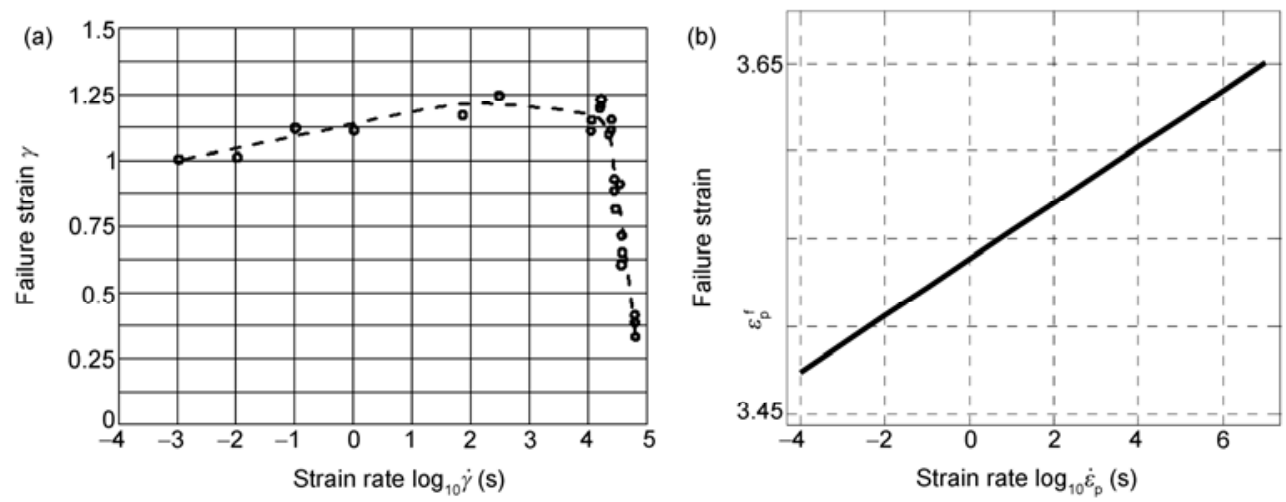

图 4 失效应变随应变率提高的变化

(a) XC18 钢失效应变实验测值; (b) AISI4340 钢失效应变计算值

徐永波和白以龙 ${ }^{[35]}$ 、王敏杰 ${ }^{[36]}$ 、Hartley 等人 ${ }^{[37]}$ 的研 究表明：由于剪切失稳使塑性变形集中在局部范围 内, 当材料产生同样宏观变形时, 集中剪切带内应变 要比均匀变形条件时变形区内的应变大, 这常导致 材料沿热塑性剪切失稳形成的集中变形带断裂, 此 时，热塑性失稳成为断裂的先导，同时，材料集中剪 切带中断裂也加速了材料失效过程. 而在高速冲击 加载下, 材料变形区首先形核产生大量微小裂纹, 微 小裂纹快速长大、连接而形成宏观断裂, 使材料突发 性失效，材料断裂失效前不再有热塑性剪切失稳形 成的集中滑移带 ${ }^{[38,39]}$.

表现在切削加工过程中, 不同的变形速度阶段 剪切失稳机制不同，导致不同切削速度阶段切屑的 形态不同, 如图 5. 随切削速度提高, 变形区材料会 因强度升高、散热条件变差而塑性增强, 沿剪切面的 剪切变形首先因热软化大于应变硬化和应变率硬化 而失稳, 即绝热剪切失稳. 这类锯齿形切屑的集中剪 切带是局部位错滑移带, 即所谓“白层”, [40]. 当切削速 度提高到一定程度, 剪切面材料塑性迅速降低, 剪切 面材料在断裂前再产生明显的塑性滑移, 剪切面材 料因断裂而变形失稳. 这类锯齿形切屑的集中剪切 带中将有明显的断裂现象, 如 Shaw 和 Komanduri 等 人 $^{[8,12]}$ 在锯齿中发现的断裂. 当切削速度进一步提高, 材料高度脆化, 材料在断裂前来不及进行塑性变形 就以脆性断裂形式破坏, 切屑形态将为崩碎切屑.

\section{2 超高速切削实验}

为了获得较宽切削速度范围内的切屑, 验证不

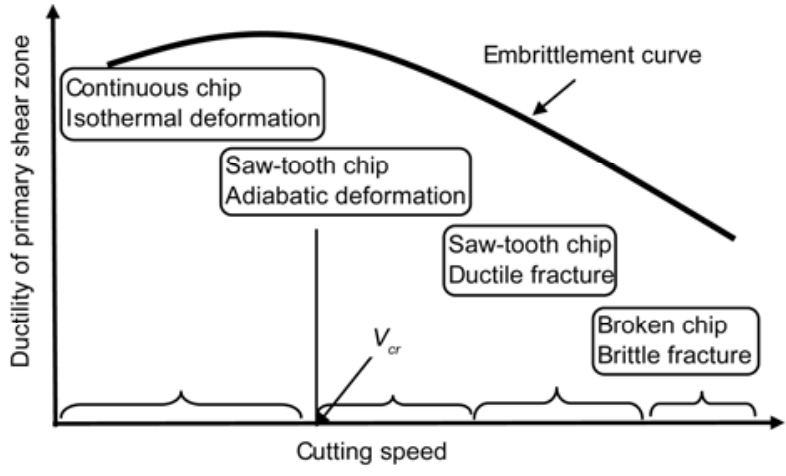

图 5 随切削速度提高剪切面材料塑性和切屑锯齿化机理 的改变

同切削速度下工件材料的脆化及这种脆化对切屑形 态的影响，设计了本超高速切削实验。实验中以 40 7000 m/min 的切削速度对 3 种不同性质的工件进 行高速直角切削, 获得不同切削速度下切屑并进行 金相学观察. 具体实验方案如下.

\section{1 工件材料}

选择 Inconel718, AerMet100, 7050-T7451 作为实 验材料. Inconel718 为镍基高温合金, 在较高温度下 仍能保持较高的强度. AerMet100 是一种钴镍类钢, 其强度高、韧性高，具有良好的综合性能. Inconel718 与 AerMet100 都属于难加工材料, 切削加工时容易产 生锯齿形切屑. 7050-T7451 是 Al-Zn-Mg-Zr 系铝合金, 具有较高的结构强度、断裂韧性和抗应力腐蚀断裂等 良好综合性能，易于进行切削加工. 3 种材料的主要 力学、物理性能见表 2 . 
表 23 种实验材料的常用力学物理性能 ${ }^{[41 ~ 45]}$

\begin{tabular}{|c|c|c|c|c|c|c|c|}
\hline Material & $\begin{array}{l}\text { Density } \\
\rho\left(\mathrm{g} / \mathrm{cm}^{3}\right)\end{array}$ & $\begin{array}{c}\text { Thermal } \\
\text { conductivity } \\
K(\mathrm{~W} / \mathrm{m} \mathrm{K})\end{array}$ & $\begin{array}{l}\text { Specific heat } \\
c(\mathrm{~J} / \mathrm{Kg} \mathrm{K})\end{array}$ & $\begin{array}{c}\text { Elastic } \\
\text { modulus } \\
E(\mathrm{GPa})\end{array}$ & $\begin{array}{c}\text { Yield } \\
\text { strength } \\
\sigma_{s}(\mathrm{MPa})\end{array}$ & $\begin{array}{c}\text { Tensile } \\
\text { strength } \\
\sigma_{b}(\mathrm{MPa})\end{array}$ & $\begin{array}{c}\text { Melting } \\
\text { temperature } \\
\operatorname{Tm}\left({ }^{\circ} \mathrm{C}\right)\end{array}$ \\
\hline Inconel718 $8^{[41,42]}$ & 8.24 & 10.63 & 435 & 200 & 1200 & 1580 & $1260 \sim 1336$ \\
\hline AerMet $100^{[43,44]}$ & 7.89 & 43 & 460 & 194.6 & 1758 & 1965 & 1464 \\
\hline $7050-\mathrm{T} 7451^{[45]}$ & 2.83 & 157 & 860 & 70.3 & 455 & 510 & $490 \sim 630$ \\
\hline
\end{tabular}

\section{2 实验方案}

对 3 种工件材料采用外圆直角切削, 实验方案如 图 6. 图中工件为厚 $2 \mathrm{~mm}$ 的圆盘, 工件通过螺钉固 定在一根轴上并将该轴装夹在机床主轴上. 采用车 削刀杆, 刀杆型号为 KENNAMETA NSR252M3. 采 用 PVD TiAIN 涂层硬质合金刀片, 其型号为 KENNAMETA NG3142R. 工件、刀具装夹后的现场 照片如图 6 右下角照片. 切削加工参数如表 3 .

收集各切削速度下的切屑并对其进行镶嵌、磨 抛、腐蚀, 然后进行光学和扫描电镜 (Scanning Electron Microscope, 简称 SEM) 观察.

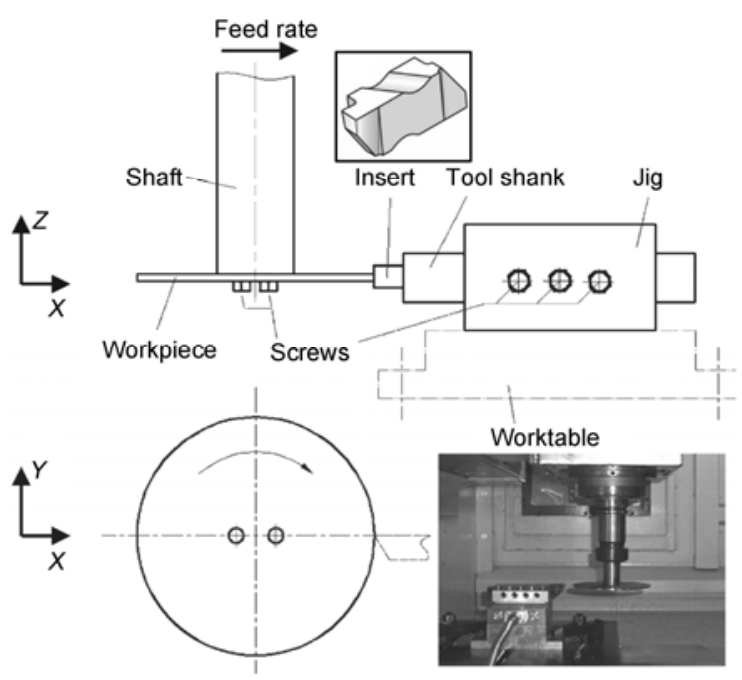

图 6 超高速切削实验方案

\section{表 3 高速直角切削实验切削参数}

\begin{tabular}{ccccc}
\hline $\begin{array}{c}\text { Cutting } \\
\text { speed } \\
V(\mathrm{~m} / \mathrm{min})\end{array}$ & $\begin{array}{c}\text { Feed per } \\
\text { tooth } \\
f(\mathrm{~mm} / \mathrm{z})\end{array}$ & $\begin{array}{c}\text { Cutting } \\
\text { width } \\
w(\mathrm{~mm})\end{array}$ & $\begin{array}{c}\text { Rake } \\
\text { angle } \\
\gamma_{0}\left({ }^{\circ}\right)\end{array}$ & $\begin{array}{c}\text { Cutting } \\
\text { fluid }\end{array}$ \\
\hline $40 \sim 7000$ & 0.1 & 2 & -20 & dry cutting \\
\hline
\end{tabular}

\section{3 实验结果分析和讨论}

\section{1 切屑形态的变化}

表 4 给出了 3 种工件材料在切削速度为 $40 \sim 7000$ $\mathrm{m} / \mathrm{min}$ 速度范围内的切屑. Inconel718 和 AerMet100 切屑同在切削速度为 $40 \mathrm{~m} / \mathrm{min}$ 时发生由带状向锯齿 形的转变, 在切削速度分别为 $1500 \mathrm{~m} / \mathrm{min}$ 和 1100 $\mathrm{m} / \mathrm{min}$ 时出现了锯齿分节分离的单元切屑. 随切削速 度进一步提高, 两种材料的切屑进一步碎化, 在 $7000 \mathrm{~m} / \mathrm{min}$ 时 AerMet100 的切屑比 Inconel718 的切 屑更加细碎：AerMet100 的切屑长度范围在 50 100 $\mu \mathrm{m}$, 而 Inconel718 的切屑长度在 200 250 $\mu \mathrm{m}$ 之间. 7050-T7451 的切屑在 $300 \mathrm{~m} / \mathrm{min}$ 时出现从带状向锯 齿形的转变, 在 $3000 \mathrm{~m} / \mathrm{min}$ 时形成了锯齿分节完全 分离的单元切屑, 随切削速度进一步提高到, 切屑没 有明显碎化.

\section{2 集中剪切带的变化}

Inconel718, AerMet100, 7050-T7451 的切屑形态 分别在 $40 \mathrm{~m} / \mathrm{min}, 40 \mathrm{~m} / \mathrm{min}, 300 \mathrm{~m} / \mathrm{min}$ 时发生了由带 状向锯齿形的转变(表 4). 切屑锯齿化临界状态时, 集中剪切带都是由局部位错滑移带组成, 集中剪切 带中没有断裂. 但随切削速度提高, 3 种材料集中剪 切带中出现断裂, 并且断裂随切削速度提高逐渐发 展. 图 7 给出在两种切削速度下 AerMet00 钢切屑集 中剪切带内的断裂状况. 可以看出, 切削速度在 100 $\mathrm{m} / \mathrm{min}$ 时集中剪切带中还未出现断裂(图 7(a)), 在 500 $\mathrm{m} / \mathrm{min}$ 时集中剪切带内的断裂已十分明显(图 7(b)). 随切削速度提高集中剪切带断裂是一个普遍现象,

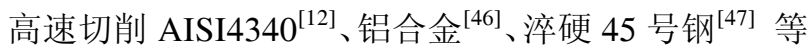
材料时都有该现象发生.

为定量说明集中剪切带中断裂随切削速度的变 化, 定义相对断裂长度 $\mathrm{Cr}$ 为 
表 43 种工件材料切屑形态转变节点及对应切削速度

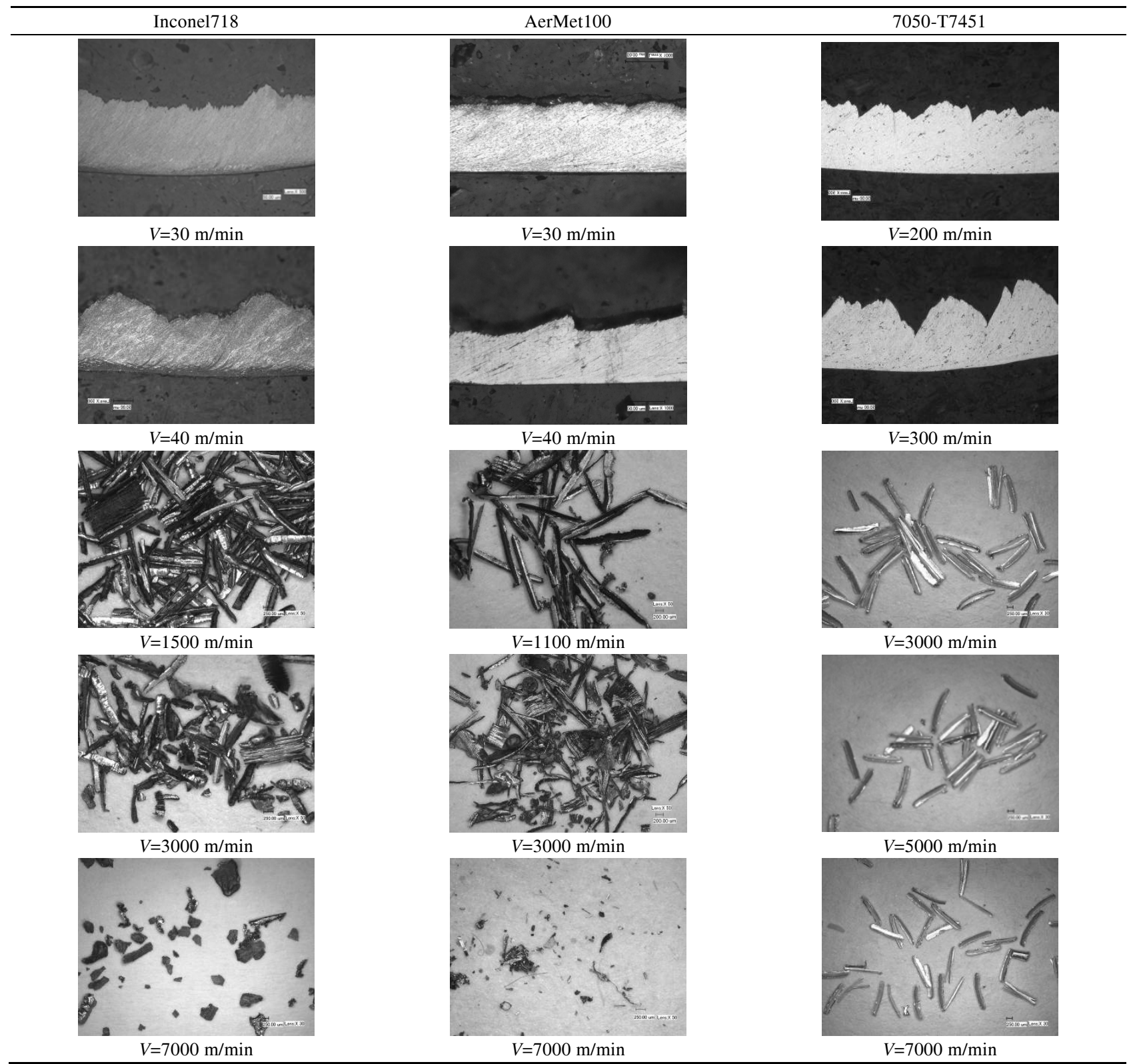

$$
C r=\frac{L_{1}}{L_{2}},
$$

式中, $L_{1}$ 是集中剪切带内断裂段长度, $L_{2}$ 是整个集中 剪切带长度(图 7(b)). Cr =0 表示切屑集中剪切带中 还未出现断裂, 而 $C r=1$ 表示形成了锯齿分节完全分 离的单元切屑. 3 种材料切屑集中剪切带的相对断裂 长度随切削速度提高的变化如图 8 所示. 从图 8 中可 以看出, 对于 Inconel718, AerMet100, 在切屑锯齿化 临界切削速度 $(V=40 \mathrm{~m} / \mathrm{min})$, 两种材料锯齿形切屑的
相对断裂长度 $C r$ 都为零. 随切削速度提高, 两种材 料切屑的对应的 $C r$ 迅速提高. 在切削速度为 200 $\mathrm{m} / \mathrm{min}$ 时, Inconel718, AerMet100 切屑对应的 $C r$ 分别 达到 $0.8,0.4$. 随后, 随切削速度进一步提高, 两种材 料切屑对应的 $C r$ 升高速度变缓. 切削速度到达 1500 $\mathrm{m} / \mathrm{min}$ 时, 两种材料切屑对应的 $C r$ 达到 1 , 即形成锯 齿切屑分节完全分离的单元切屑. 7050-T7451 在 200 $\mathrm{m} / \mathrm{min}$ 时 $C r=0$, 随后 $C r$ 随切削速度缓慢上升, 在 $2000 \mathrm{~m} / \mathrm{min}$ 后 $C r$ 快速上升, 在 $3000 \mathrm{~m} / \mathrm{min}$ 时 $C r=1$, 

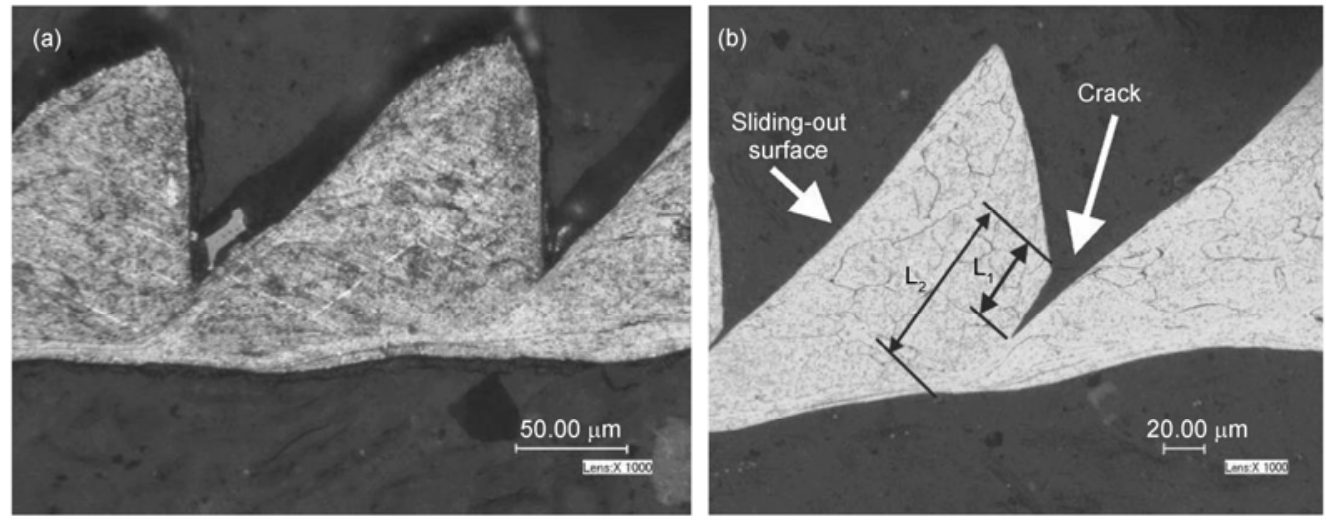

图 7 AerMet100 钢在两种切削速度下集中剪切带的对比

(a) $V=100 \mathrm{~m} / \mathrm{min}$; (b) $V=500 \mathrm{~m} / \mathrm{min}$

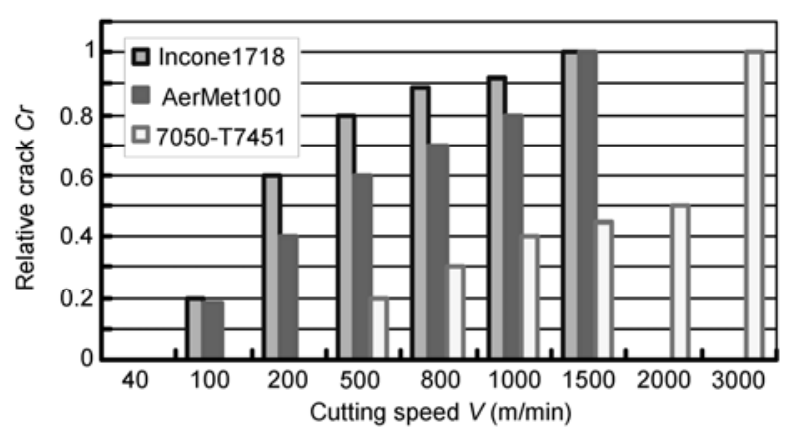

图 83 种工件材料集中剪切带断裂随切削速度的变化

即形成锯齿切屑分节完全分离的单元切屑.

\section{3 切屑滑出面的变化}

锯齿切屑分节滑出面如图 7(b) 所示. 在不同的切 削速度切屑滑出面也具有不同的形貌, 如图 9. 从图 9(a)中可以观察到在切削速度为 $100 \mathrm{~m} / \mathrm{min}$ 时, 滑出 面内出现细长的沟槽, 这些沟槽是锯齿分节在剪切 滑移过程时因硬质颗粒耕犁而产生或是被拉长的孔 洞. 在 $500 \mathrm{~m} / \mathrm{min}$ 时(图 9(b)), 滑出面内拉伸形成的孔 洞增多. 在 $1000 \mathrm{~m} / \mathrm{min}$ 时, 剪切面内出现了大量的韧 窝(图 9(c)), 表明在此切削速度下锯齿分节滑出时发 生了韧性断裂.

\section{4 工件材料的脆化}

随切削速度提高, 切屑碎化(表 4)、集中剪切带 中断裂加剧(图 8)和切屑滑出面的韧性断裂增多(图 9) 的现象说明了工件材料逐渐变脆的过程. 从滑出面 的形貌看(图 9), 在形成锯齿分节分离的单元切屑时
发生的是韧性断裂, 说明此时材料仍具备一定的塑 性. 将在 $7000 \mathrm{~m} / \mathrm{min}$ 时获得的切屑进行局部放大, 如 图 10. 图 10 显示 AerMet100 切屑形成时发生脆性断 裂. Inconel718 的切屑也有脆性断裂的形貌, 但不如 AerMet100 明显. 7050-T7451 切屑出现部分脆性断裂. 说明在 $7000 \mathrm{~m} / \mathrm{min}$ 切削时 Inconel718, AerMet100 切 屑材料已完全脆化, 切屑变形以脆性断裂而破坏, 形 成崩碎切屑; 7050-T7451 虽脆性加剧, 但还未到完全 脆化的程度.

如在 2.3 中所分析, 不同应变率阶段材料塑性变 形控制机理不同，导致不同切削速度阶段切屑形成 机理不同, 如在切屑锯齿化临界切削速度, 出现了因 热塑性剪切失稳产生的锯齿形切屑(表 $4, V=40 \mathrm{~m} / \mathrm{min}$ 时 Inconel718, AerMet100 切屑; $V=300 \mathrm{~m} / \mathrm{min}$ 时 7050-T7451 切屑), 该类锯齿形切屑的集中剪切带为 局部塑性滑移带, 滑移带中没有断裂. 在更高切削速 度范围, 材料塑性降低, 锯齿形切屑产生机理过渡为 周期性韧性断裂, 切屑集中剪切带中出现韧性断裂, 如 AerMet100 钢在 $1000 \mathrm{~m} / \mathrm{min}$ 时切屑(图 9). 当切削 速度高到足以使材料高度脆化时, 切屑变形为脆性 断裂破坏, 形成崩碎状切屑(图 10).

\section{4 基于切屑形态的切削速度阶段划分方法}

从以上研究可知, 对同一材料切屑形态可用来 表示所用切削速度的范围, 即形态切屑与切削速度 范围有对应关系, 如带状切屑对应的切削速度要低 于锯齿形切屑对应的切削速度，而锯齿形切屑对应 

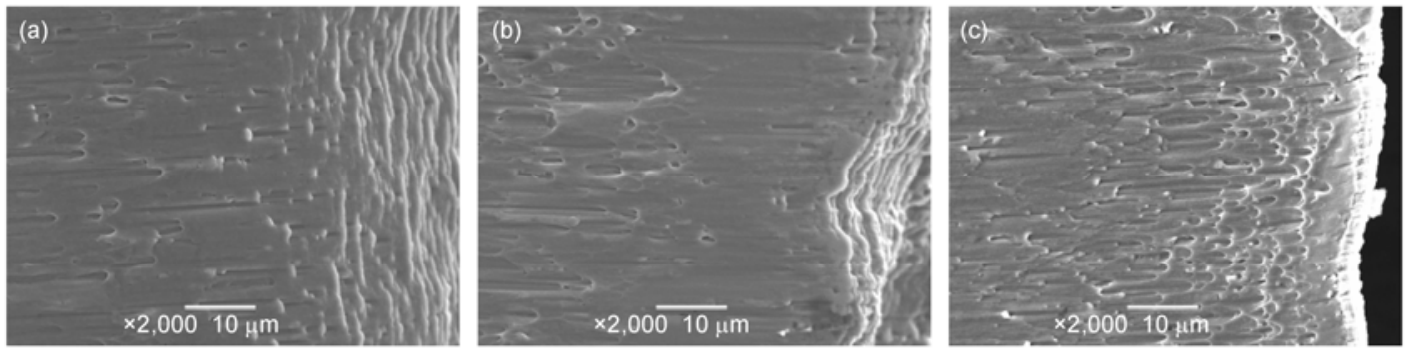

图 93 种切削速度下 AerMet100 钢切屑滑出面形貌

(a) $V=100 \mathrm{~m} / \mathrm{min}$; (b) $V=500 \mathrm{~m} / \mathrm{min}$; (c) $V=1000 \mathrm{~m} / \mathrm{min}$
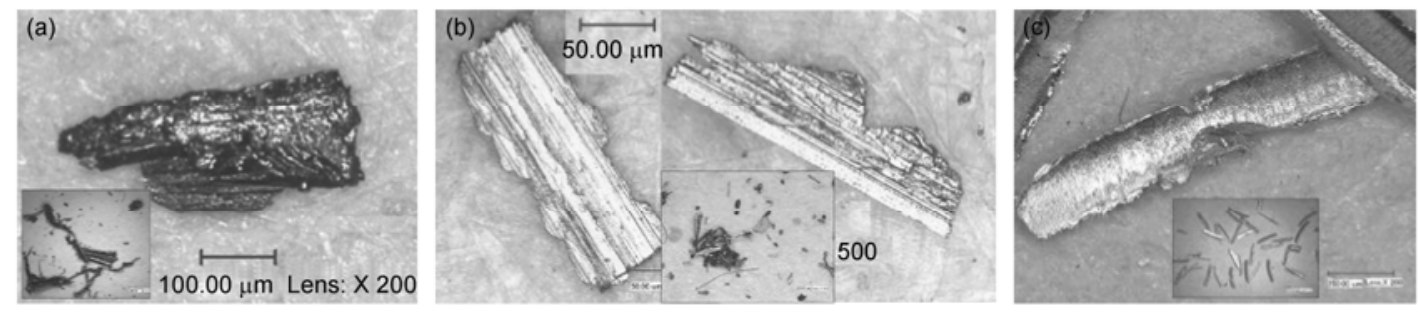

图 103 种工件材料在 $7000 \mathrm{~m} / \mathrm{min}$ 时的切屑形态

(a) Inconel718; (b) AerMet100; (c) 7050-T7451

的切削速度又低于锯齿分节分离单元切屑对应的切 削速度, 因此可以从切屑形态的角度对切削速度阶 段进行划分. 根据不同切削速度阶段切屑形态的不 同, 按照从低到高的顺序将可将切削速度划分为 3 个 阶段.

1) 普通切削速度阶段：即低于形成锯齿形切屑 的切削速度. 在该切削速度范围, 材料塑性较好, 切 屑变形区材料因应变硬化大于热软化, 切屑内发生 的是均匀的等温塑性变形, 切屑呈连续带状.

2) 高速切削速度阶段: 即从开始形成锯齿形切 屑到锯齿形切屑分节完全分离的切削速度范围. 在 该切削速度范围, 材料强度随切削速度提高而升高, 同时材料塑性逐渐降低, 切屑分节内变形较少, 变形 主要集中在集中剪切带内, 切屑锯齿化机制由热塑 性剪切失稳逐渐过渡到韧性断裂失效(或称周期性韧 性断裂), 切屑呈锯齿状.

3) 超高速切削速度阶段: 即形成分离锯齿形切 屑分节(单元切屑)至更高速度范围的切削速度. 在该 切削速度范围材料塑性迅速降低、脆性迅速增加, 切 屑变形逐渐由周期性韧性断裂过渡为均匀性脆性破 坏, 切屑逐渐由分离的锯齿分节过渡到崩碎状碎屑.

按照以上切削速度阶段划分方法, 对本文研究 所用 3 种工件材料进行切削速度阶段划分, 结果如表
5. 表 5 表明 Inconel718 和 AerMet100 钢具有相同的 切削速度分段, 高速切削速度范围都在 40 1500 $\mathrm{m} / \mathrm{min}$, 超过 $1500 \mathrm{~m} / \mathrm{min}$ 后进入超高速切削速度阶段; 7050-T7451 铝合金从 $3000 \mathrm{~m} / \mathrm{min}$ 开始出现分离的锯 齿分节切屑, 意味着 7050-T7451 铝合金从 3000 $\mathrm{m} / \mathrm{min}$ 开始进入超高速切削速度阶段.

需要指出, 切屑形态取决于工件材料流经第一 变形区时的动态力学性能(强度和塑脆性)的演化, 而 影响材料流经第一变形区时动态力学性能的因素不 仅有切削速度, 还有如刀具前角、切削刃口圆弧半 径、切削厚度等参数的影响.

\section{5 结论}

本文分析了随切削速度提高工件材料力学性能 (强度和塑脆性)的变化规律, 探索了这种变化对切屑 锯齿化的影响机制, 揭示了不同切削速度阶段切屑 形态形成的控制机理, 并通过高速直角切削实验进 行了验证, 提出了基于切屑形态的切削速度阶段划 分方法. 具体研究结论可以总结如下.

1) 随切削速度(变形速度)提高, 塑性变形需要 更多的位错源同时开动，材料的临界屈服应力因此 而增大. 塑性变形发动后, 材料的流动应力由材料应 


\section{表 53 种材料切削速度阶段划分}

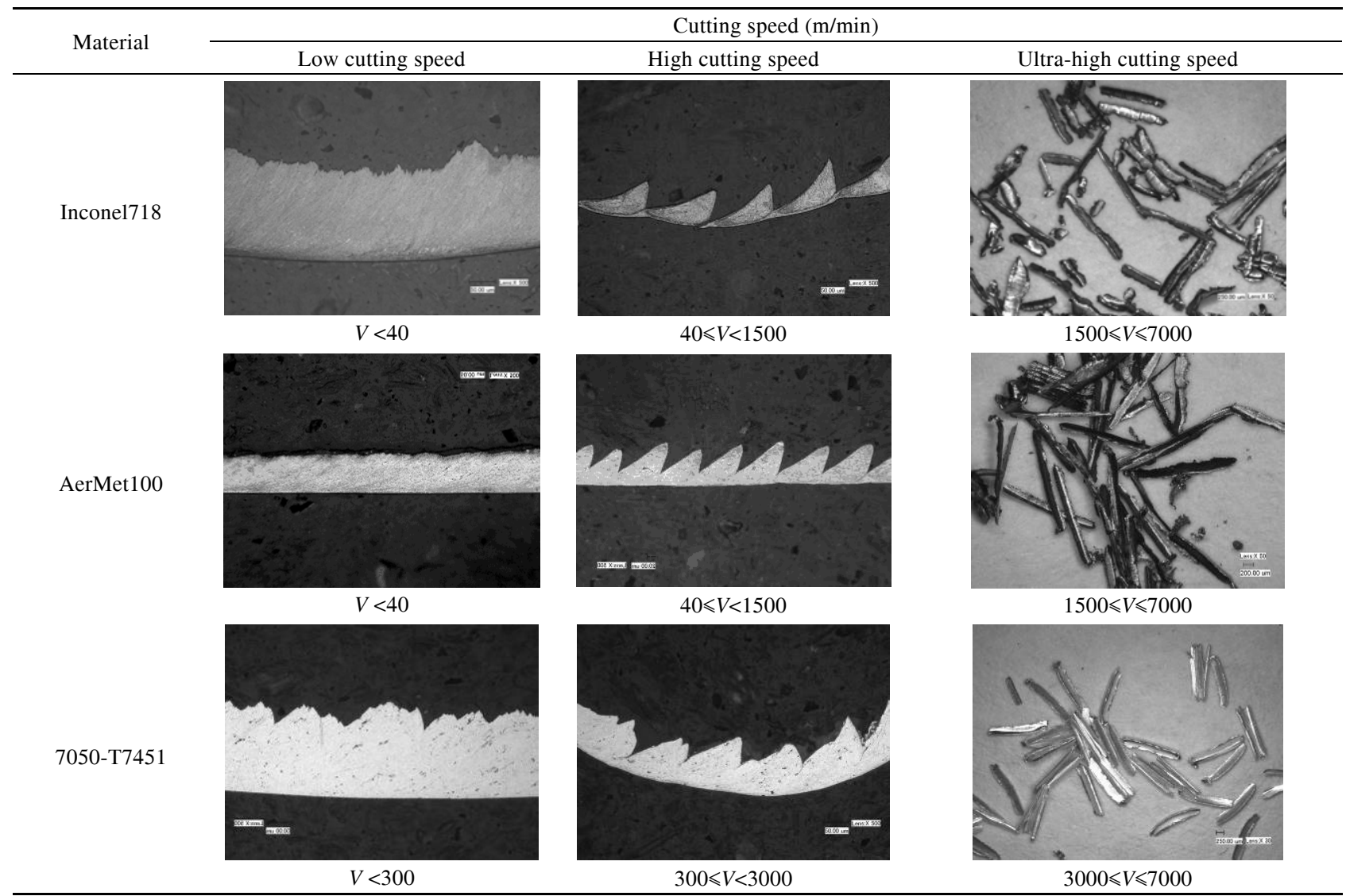

变硬化、应变率硬化和塑性变形做功引起的热软化共 同决定. 切削过程中, 较高的材料流动应力使得第一 变形区材料在产生同样应变时获得更高的温升和更 明显的热软化效应，从而导致变形区工件材料在更 小的应变处发生热塑性剪切失稳, 促使锯齿形切屑 的产生.

2) 随切削速度(变形速度)提高, 变形区材料温 度升高, 在扩散蠕变控制塑性变形阶段, 变形部位材 料迅速发生恢复再结晶, 使材料的塑性得到提高. 但 在阻尼机制控阶段，位错来不及堆积和滑移，位错的 移动以脆性断裂的形式进行. 材料脆性增加, 可使切 削层工件材料流经第一变形区时还未产生明显热软 化就发生断裂失效, 剪切面变形抗力降低并促使锯 齿形切屑产生.
3) 由于不同应变率阶段的塑性变形机制不同, 随切削速度提高, 变形区工件材料将先因热软化效 应大于应变硬化和应变率硬化效应而发生热塑性剪 切失稳. 然后, 在更高的切削速度范围会发生因断裂 失效导致的剪切失稳. 对应这两种情况下产生锯齿 形切屑的控制机理分别是热塑性剪切失稳和周期性 韧性断裂失效. 当切削速度高到足以使材料完全脆 化时, 切屑因脆性断裂而破坏, 此时切屑为崩碎切 屑.

4) 切屑形态与切削速度范围存在对应关系，根 据切屑形态可将切削速度划分为普通切削速度阶段、 高速切削速度阶段、超高速切削速度阶段, 相应的切 屑形态分别为带状切削、锯齿形切屑、单元切屑(包 括崩碎切屑). 
参考文献

1 周泽华. 金属切削理论. 北京: 机械工业出版社, 1992

2 Komanduri R, Brown R H. On the mechanics of chip segmentation in machining. J Eng Ind, 1981, 103: 33-51

3 Trent E M, Wright P K. Metal Cutting. Massachusettes: Butterworth-Heinemann, 2000

4 Shaw M C. Metal Cutting Principles. Oxford: Clarendon Press, 1984

5 Astakhov V P. Tribology of Metal Cutting. London: Elsevier, 2006

6 Nakayama K, Arai M, Kanda T. Machining characteristics of hard materials. Ann CIRP, 1988, 37(1): 89-92

7 Shaw M C, Vyas A. Chip formation in the machining of hardened steel. Ann CIRP, 1993, 42(1): 29-33

8 Vyas A, Shaw M C. Mechanics of saw-tooth chip formation in metal cutting. J Manuf Sci Eng, 1999, 121(2): 163-172

9 Su G S, Liu Z Q. An experimental study on influences of material brittleness on chip morphology. Int J Adv Manuf Technol, 2010, 51: $87-92$

10 Elbestawi M A, Srivastava A K, El-Wardany T I. A model for chip formation during machining of hardened steel. Ann CIRP, 1996, 45(7): 71-76

11 Davies M A, Chou Y, Evans C J. On the chip morphology, tool wear and cutting mechanics in finish hard turning. Ann CIRP, 1996, 45(1): $77-82$

12 Komanduri R, Schroeder T, Hazra J, et a1. On the catastrophic shear instability in high-speed machining of an AISI 4340 steel. J Eng Ind, 1982, 104: 121-131

13 Gente A, Hoffmeister H W. Chip formation in machining Ti-6Al-4V at extremely high cutting speeds. Ann CIRP, 2001, 50(1): 49-52

14 Oxley P L B. Mechanics of Machining: An Analytical Approach to Assessing Machinability. Chichister: Ellis Horwood, 1989

15 Hargreaves C R, Werner L. Adiabatic Shear-An Annotated Bibliography. Report No. AD/A006490, National Technical Information Service, Washington, DC, 1974

16 Băker M, Rösler J, Siemers C. The influence of thermal conductivity on segmented chip formation. Comput Mater Sci, 2003, 26: 175-182

17 Băker M. The influence of plastic properties on chip formation. Comput Mater Sci, 2003, 28: 556-562

18 Liu Z Q, Su G S. Characteristics of chip evolution with elevating cutting speed from low to very high. Int J Mach Tool Manu, 2012, 54-55: $82-85$

19 段春争. 正交切削高强度钢绝热剪切行为的微观机理研究. 博士学位论文. 大连: 大连理工大学, 2005

20 周惠久, 黄明志. 金属材料强度学. 北京: 科学出版社, 1983

21 Armstrong R W, Werner A, Zerilli F J. Dislocation mechanics of iron and copper in high rate deformation tests. In: Elert M, Furnish M D, Anderson W W, et al., eds. Proceedings of American Institute of Physics Conference. Shock Compression of Condensed Matter 2009, Nashville. Vol. 1195. 2009. 1105-1108

22 Clifton R. Dynamic plasticity. J Appl Mech, 1983, 50: 941-952

23 毛卫民, 朱景川, 酣剑, 等. 金属材料结构与性能. 北京: 清华大学出版社, 2008

24 刘战强, 吴继华, 史振宇, 等. 金属切削变形本构方程的研究. 工具技术, 2008, 42: 3-9

25 Johnson G R, Cook W H. A constitutive model and data for metals subjected to large strains, high strain rates and high temperature. In: Proceedings of the 7th International Symposium on Ballistics. Volum 547(11). Netherlands: The Hague, 1983. 541-547

26 Uhlmann E, Schulenburg M G, Zettier R. Finite element modeling and cutting simulation of Inconel 718. Ann CIRP, 2007, 56(1): 61-64

27 Klepaczko J R. Plastic shearing at high and very high strain rates. J Phys III, 1994, 4: 35-40

28 Armstrong R W, Walley S M. High strain rate properties of metals and alloys. Int Mater Rev, 2008, 53(3): 105-128

29 Zerilli F J, Armstrong R W. Dislocation mechanics based constitutive relations for material dynamics calculations. J Appl Phys, 1987, 61(5): $1816-1825$

30 Krajcinovic D, Mastilovic S, Vujosevic M. Brittle to quasi-brittle transition. Meccanica, 1998, 33: 363-379

31 Johnson G R, Cook W H. Fracture characteristics of three metals subjected to various strains, strain rates, temperatures, and pressures. Eng Frac Mech, 1985, 21: 31-48

32 Mabrouki T, Rigal J F. A contribution to a qualitative understanding of thermo-mechanical effects during chip formation in hard turning. J Mater Process Technol, 2006, 176: 214-221

33 Lorentzon J, Järvstråt N, Josefson B L. Modelling chip formation of alloy 718. J Mater Process Technol, 2009, 209(10): 4645-4653

34 Shi J, Liu C R. On predicting chip morphology and phase transformation in hard machining. Int J Adv Manuf Technol, 2006, 27: 645-654

35 徐永波, 白以龙. 动态载荷下剪切变形局部化、微结构演化与剪切断裂研究进展. 力学进展, 2007, 37(4): 496-516

36 王敏杰. 动态塑性试验技术. 力学进展, 1988, 18(1): 70-78 
37 Hartley K A, Dufy J, Hawley R H. Measurement of the temperature profile during shear band formation in steels deforming at high strain rates. J Mech Phys Solids, 1987, 35: 283-301

38 白以龙, 汪海英, 夏蒙棼, 等. 固体的统计细观力学-连接多个耦合的时空尺度. 力学进展, 2006, 36(2): 286-311

39 何国威, 夏蒙棼, 柯孚久, 等. 多尺度耦合现象: 挑战和机遇. 自然科学进展, 2004, 14(2): 121-126

40 徐永波, 白以龙. 动态载荷下剪切变形局部化、微结构演化与剪切断裂研究进展. 力学进展, 2007, 37(4): 496-516

41 Jacobsson L, Persson C, Melin S. Thermo-mechanical fatigue crack propagation experiments in Inconel718. Int J Fatigue, 2009, 31 : 1318-1326

42 Thakur D G, Ramamoorthy B, Vijayaraghavan L. Study on the machinability characteristics of superalloy Inconel718 during high speed turning. Mater Design, 2009, 30: 1718-1725

43 Lippard H E, Campbell C E, Dravid V P, et al. Microsegregation behavior during solidification and homogenization of AerMet100 steel. Metall Mater Trans B, 1998, 29(1): 205-210

44 Xu Y Q, Zhang T, Bai Y M. Effect of grinding process parameters on surface layer residual stress. Adv Mater Res, 2010, 135: 154-158

45 付秀丽, 艾兴, 万熠, 等. 铝合金 7050 高温流变应力特征及本构方程. 武汉理工大学学报, 2006, 28(12): 113-116

46 Schulz H, Abele E, Sahm A. Material aspects of chip formation in HSC machining. Ann CIRP, 2001, 50(1): 45-48

47 苏国胜, 刘战强. 基于剪切带断裂特征的锯齿形切屑形成机理演化的研究. 工具技术, 2010, 44(10): 17-19 